\title{
Grundprinzipien von Future Skills
}

Hört man den Personalverantwortlichen von Organisationen zu, die sich bereits weitgehend mit den neuen Formen von Arbeit und Governance beschäftigt haben, dann wird deutlich, dass sich alle auf dem Weg zu vernetzten und agilen Organisationen befinden. Mit durchaus unterschiedlichen Geschwindigkeiten und Ausprägungen, aber doch mit ähnlichen Ergebnissen. Welches sind die Merkmale, die bei allen gleich sind? Was wirkt im Hintergrund auf die Veränderungen? Und was kann man daraus lernen, um besser für die Zukunft vorbereitet zu sein?

Nun - es wird deutlich, dass allen bisher berichteten Vignetten und Episoden die gleiche Entwicklung zugrunde liegt: Organisationen haben sich auf den Weg gemacht, die Grenze zwischen Struktur und Dynamik weiter in Richtung Dynamik zu verschieben. Das ist ein für viele Organisationen noch weitgehend unbekanntes Terrain. Alle Interviews machen deutlich, dass wir es mit einem Zukunftsbereich der Entwicklung zu tun haben. Hier wird experimentiert und Maßnahmen ausprobiert.

Mit Blick auf die Frage, was zukünftige Mitarbeiterinnen und Mitarbeiter brauchen, um in diesen sich wandelnden Arbeitsgebieten erfolgreich handeln zu können: Fachliches, abrufbares Wissen reicht nicht mehr aus, um diese Entwicklung mitzugestalten. Es sind vielmehr Future Skills, denen vor allem Aspekte wie Selbstbewusstsein, Selbstkompetenz, Selbstwert, Autonomie und Leistungsmotivation zugrunde liegen. Dort, wo fach- und methodenbezogene Kompetenzen gefragt sind, ist es weniger traditionelles Methodenwissen, etwa der Businessanalyse oder Fachwissen in einem bestimmten Bereich, sondern es sind Kompetenzen wie Flexibilität und Offenheit, Vielseitigkeit, Fähigkeit zum Perspektivwechsel, Interdisziplinarität, Innovationskompetenzen wie beispielsweise Kreativität, Innovatives Denken, Experimentierbereitschaft, Systemkompetenzen, Systems-Thinking, Wissen über Wissensstrukturen, vernetztes Denken, analytische Kompetenz oder auch Digitalkompetenzen. 
Präsentiert man die Liste dieser Kompetenzen Personalverantwortlichen in Future Organisations (zur Definition von Future Organisations s. Kapitel A 1.3.1 Schritt 1: Identifikation von „Future Organisations“), dann wird der Wunsch laut, neben einer rein additiven Aufzählung auch die zugrundeliegenden Prinzipien herauszuarbeiten und ein Modell der Skills unter Bedingungen immer höherer Selbstorganisation in der Zukunft herauszulösen. In der Future Skills Studie ist dies ein zentrales Anliegen. Dabei fällt zunächst einmal auf: Future Skills verschieben den Fokus, weg von der Arbeit als vorgegebener, extern strukturierter Aktivität, die einem bereits vorstrukturierten Handlungsplan folgt, hin zu einer Beschäftigungsagenda, die Mitarbeiterinnen und Mitarbeiter durch eigene Beteiligung situativ mitgestalten. Mit hoher Identifikation, großer Motivation und der Möglichkeit zu eigener Strukturierung der Arbeit. Ein wesentliches und konstituierendes Element der benannten Future Skills ist die Fähigkeit zur Selbstorganisation. Selbstorganisation weist auf die besondere Bedeutung hin, die dem Subjekt als dem Gestaltenden zukommt. Für Organisationen, die per Definition aus verbindlichen Strukturen bestehen, bedeutet dies, ein Spannungsverhältnis einzugehen. Je mehr sie sich in agilen und unvorhersehbaren Arbeitsgebieten befinden - wie alle diejenigen Organisationen, die an der Future Skills Studie teilgenommen haben - desto mehr müssen sie in diesem Sinne Spannungsfeldmanagement betreiben. Es besteht darin, über Kommunikations- und Beteiligungsvorgänge Strukturen aufzubauen, in denen die Organisationsmitglieder die Strukturen, in denen sie arbeiten gleichzeitig hinterfragen, mitaushandeln und bestimmen können, ohne eine übergreifende Verbindlichkeit und Kohärenz, Erwartbarkeit und Kalkulierbarkeit zu verlieren. Wir nennen dieses Spannungsverhältnis auch das Struktur-Innovations-Paradoxon. Dabei besteht das Paradoxe darin, dass Organisationen im innersten durch Strukturen definiert sind, die auch die innere Verbindlichkeit und Erwartbarkeit für ihre Mitglieder ausmachen. Paradoxerweise hängt die Zukunftsfähigkeit von Organisationen immer stärker davon ab, diese Strukturen zu hinterfragen und passende, neue und innovative Strukturen aufzubauen. In diesem paradoxen Spannungsfeld agieren heutige Führungskräfte. Sie sind damit konfrontiert, genau diese Kompetenzen aufzubauen und selbst zu praktizieren - den Umgang mit diesem Spannungsfeld. Dieses Verständnis drückt sich in mehr Wert- und weniger Regelorientierung aus. Mehr Kommunikations- und weniger Strukturorientierung. Es geht um den Aufbau und die Weiterentwicklung von Organisationkulturen, die sich dynamisch weiterentwickeln.

Organisationen, die beginnen, sich auf diese Weise auszurichten, benötigen nicht nur spezifische Strukturen, sondern bedürfen auch eines besonderen Verständnisses davon, wie Lernen und Entwickeln innerhalb der Organisationen funktioniert. Denn Lernen und Entwickeln wird zu einer Grundkonstituente solcher Organisa- 
tionen. Es bestimmt über die Fähigkeit der Organisationmitglieder und damit auch der Gesamtorganisation, sie den zukünftigen Erfordernissen in geeigneter Weise anzupassen. Die NextSkills Studie zeigt, dass die Befragten alle der vier folgenden Bereiche explizit benennen und elaboriert ausführen können:

1. Die aus ihrer Sicht wichtigsten Future Skills,

2. die notwendigen Führungskompetenzen für das beschriebene Spannungsfeldmanagement, und

3. die organisationalen Lernansätze. Und - alle haben

4. dezidierte Anforderungen, die sich daraus ergeben, wie Hochschulausbildung gestaltet sein muss.

Das Schlagwort heißt dabei: Selbstorganisation ermöglichen. Und damit sind wir wieder beim Stichwort, das dieses Kapitel umrahmt und mit dem es angefangen hat. Aus den bisherigen Analysen und den Berichten der Teilnehmer an der NextSkills Studie schälen sich neun verschiedene Prinzipien heraus, die für das Konzept der Future Skills von Bedeutung sind und nachfolgend jeweils erläutert werden.

\section{Prinzip 1: Organisationen sind Teil von vernetzten, systemischen Umwelten}

Organisationen, die in ihnen handelnden Akteurinnen und Akteure und die Umwelt hängen als vernetzte sich gegenseitig beeinflussende Teilsysteme miteinander zusammen. Die Veränderungen der globalen Umwelt, der Organisationen und die handelnden Subjekte sind systemisch so miteinander verknüpft, dass sie ein gemeinsames Ökosystem bilden: Megatrends der demografischen Veränderung, der Globalisierung und Digitalisierung führen zu komplexeren, vernetzteren Umweltkontexten, die bedingen, dass auch innerhalb von Organisationen der Druck steigt, vernetzte und komplexe Strukturen auszubilden. Gemäß des kybernetischen Gesetztes von Ashby (1974) können Organisationen vor allem dann mit komplexen Umweltveränderungen umgehen, wenn sie in deren innerer Struktur komplexe Handlungsstrukturen ermöglichen können (siehe Kapitel II Der Future Skills Turn ). Auch für die handelnden Subjekte ändert sich die Lage, denn sie müssen in diesen Strukturen handlungsfähig bleiben, d.h., sie sehen sich neuen Qualifikationsanforderungen gegenüber.

\section{Prinzip 2: Organisationen streben in ein homöostatisches Gleichgewicht}

Der Begriff sowie das Konzept der Homöostase wurde ca. 1860 von Claude Bernard beschrieben. Später griffen Walter Cannon und von Karl Ludwig von Bertalanffy den Begriff auf und entwickelten seine Bezeichnung 1929 und 1932 weiter (zitiert 
nach Flechtner 1972). Er bezeichnet die Aufrechterhaltung eines Gleichgewichtszustandes eines offenen dynamischen Systems durch einen internen regelnden Prozess. Homöostase ist damit ein Sonderfall der Selbstregulation von Systemen.

Ein System, welches ein anderes steuert, kann umso mehr Störungen in dem Steuerungsprozess ausgleichen, desto größer seine Handlungsvarietät ist: Je größer die Varietät eines Systems, desto mehr kann es die Varietät seiner Umwelt durch Steuerung vermindern (siehe Ashbys Law 1974). Daraus folgt, dass die Varietät des Steuerungssystems mindestens ebenso groß sein muss wie die Varietät der auftretenden Störungen, damit es die Steuerung ausführen kann. Konkret: Wann immer es darum geht, erfolgreich mit hoch komplexen und dynamischen Situationen umzugehen, muss das handelnde System mindestens die gleiche Komplexität und Dynamik aufweisen wie das (Umwelt)System, in dem gehandelt wird. Wenn der Markt sich immer weiter vernetzt, wird es immer wichtiger, freie Vernetzung auch in den Organisationen zuzulassen und zu fördern.

\section{Prinzip 3: Selbstorganisation als Voraussetzung für Handlungsfähigkeit}

Selbstorganisation wird zu einer Schlüsselkategorie für Handlungsfähigkeit unter sich stetig wandelnden Bedingungen. Organisationen können nur agil handlungsfähig bleiben, wenn Mitarbeiterinnen und Mitarbeiter in der Lage sind, eigene Ordnungsmuster für die sie betreffenden Kontexte herauszubilden. Selbstorganisation in diesem Sinne wird verstanden als eine Kompetenz, die zu erlernen ist. Sie bildet gleichzeitig ein zentrales Prinzip, als Metakonzept für das Verstehen der Entstehung, Aufrechterhaltung und Entwicklung von Ordnungsmustern. (siehe auf Kapitel B 2.1 „Drift to Self-Organisation“: Selbstorganisation als gesellschaftliches Leitprinzip)

\section{Prinzip 4: Ermöglichende Organisationsstrukturen}

Im Anschluss an die Selbstorganisationskompetenz geht es auch darum, Organisationsstrukturen so zu gestalten, dass deren Aufbau ermöglicht wird. In Organisationen geht es zunehmend darum, Rahmenbedingungen zu gestalten, d.h. die Ökologie, in der Lösungen für Problemstellungen heranwachsen, in der in einer Forschungs- und Entwicklungsökologie neue Produkte entstehen, als nichtdeterministisch steuerbare Prozesse zu begreifen (siehe Kapitel B 4 Future Skills für Future Organisationen: Analyse zukünftiger Organisationsmodelle).

\section{Prinzip 5: Vom (Fach)wissen zur Handlungskompetenz}

Es geht um Kompetenz nicht um Wissen, also um Handlungsfähigkeit, die weitergeht als bloßes Wissen oder Erkenntnis. Es soll etwas entschieden, umgesetzt, vorangebracht werden. 


\section{Prinzip 6: Individualisierung und Personalisierung von Lernen und Entwicklung}

Lernwege werden individualisierter und personalisierter: Was, wann, wo und wie gelernt wird, bestimmt sich anhand von individuellen Lernbedürfnissen, die sich aus einem individuellen Handlungsdruck ergeben.

\section{Prinzip 7: Umgang mit Ambiguität und Unsicherheit als Kernkompetenz}

Es geht um Handlungsfähigkeit in prinzipiell offenen Situationen, also in Situationen von Unsicherheit oder Ambiguität.

\section{Prinzip 8: Lernformate für Future Skills}

Dabei stehen solche Lernformen und Formen der Unterstützung im Mittelpunkt, die eher auf aktive Begleitung abzielen als auf Belehrung oder Lehren. Immer weniger geht es um Weiterbildung und Trainings im klassischen Sinne, sondern stattdessen mehr und mehr um direkt in der Praxis wirkende Unterstützung, die einzelne Mitarbeiterinnen und Mitarbeiter konkret in ihrem professionellen Kontext unterstützt. Lernen wird nicht mehr durch klassisches Belehren gefördert, sondern durch neue Formate, die eher Mentoring, Coaching, Reflexionsbegleitung, Vernetzung oder auch das Bilden von Lerngemeinschaften umfasst. Lernen hat keine Ausbildungsfunktion im Sinne eines Erwerbs von vorgegebenen Curricula, sondern die Funktion eines stetigen Weiterentwickelns anhand konkreter Problemsituationen auf Basis von Reflexionen und dem Bilden neuer eigener Handlungsstrategien.

\section{Prinzip 9: Spanungsverhältnis von Organisationsstruktur und Selbstorganisation}

Die Organisationsstruktur der jeweiligen Organisation und das Prinzip der Selbstorganisation der jeweiligen Mitarbeiterinnen und Mitarbeiter stehen immer in einem kreativ-konstruktiven Spannungsverhältnis. Dieses gilt es produktiv in der Personalarbeit und Organisationsgestaltung aufzugreifen. 